\title{
Indirect control and power in mutual control structures
}

Citation for published version (APA):

Karos, D., \& Peters, H. J. M. (2013). Indirect control and power in mutual control structures. Maastricht University, Graduate School of Business and Economics. GSBE Research Memoranda No. 048 https://doi.org/10.26481/umagsb.2013048

Document status and date:

Published: 01/01/2013

DOI:

10.26481/umagsb.2013048

Document Version:

Publisher's PDF, also known as Version of record

\section{Please check the document version of this publication:}

- A submitted manuscript is the version of the article upon submission and before peer-review. There can be important differences between the submitted version and the official published version of record.

People interested in the research are advised to contact the author for the final version of the publication, or visit the DOI to the publisher's website.

- The final author version and the galley proof are versions of the publication after peer review.

- The final published version features the final layout of the paper including the volume, issue and page numbers.

Link to publication

\footnotetext{
General rights rights.

- You may freely distribute the URL identifying the publication in the public portal. please follow below link for the End User Agreement:

www.umlib.nl/taverne-license

Take down policy

If you believe that this document breaches copyright please contact us at:

repository@maastrichtuniversity.nl

providing details and we will investigate your claim.
}

Copyright and moral rights for the publications made accessible in the public portal are retained by the authors and/or other copyright owners and it is a condition of accessing publications that users recognise and abide by the legal requirements associated with these

- Users may download and print one copy of any publication from the public portal for the purpose of private study or research.

- You may not further distribute the material or use it for any profit-making activity or commercial gain

If the publication is distributed under the terms of Article $25 \mathrm{fa}$ of the Dutch Copyright Act, indicated by the "Taverne" license above, 


\section{Maastricht University}

Dominik Karos, Hans Peters

I ndirect control and power in mutual control structures

RM/ 13/048

\section{GSBE}

Maastricht University School of Business and Economics

Graduate School of Business and Economics

P.O Box 616

NL- 6200 MD Maastricht

The Netherlands 


\title{
Indirect control and power in mutual control structures*
}

\author{
Dominik $\operatorname{Karos}^{\dagger} \quad$ Hans Peters ${ }^{\ddagger}$
}

This version, August 2013

\begin{abstract}
In a mutual control structure agents exercise control over each other. Typical examples occur in the area of corporate governance: firms and investment companies exercise mutual control, in particular by owning each others' stocks. In this paper we formulate a general model for such situations. There is a fixed set of agents, and a mutual control structure assigns to each subset (coalition) the subset of agents controlled by that coalition. Such a mutual control structure captures direct control. We propose a procedure in order to incorporate indirect control as well: if $S$ controls $T$, and $S$ and $T$ jointly control $R$, then $S$ controls $R$ indirectly. This way, invariant mutual control structures result. Alternatively, mutual control can be described by vectors of simple games, called simple game structures, each simple game describing who controls a certain player, and also those simple games can be updated in order to capture indirect control. We show that both approaches lead to equivalent invariant structures.

In the second part of the paper, we axiomatically develop a class of power indices for invariant mutual control structures. We impose four axioms with a plausible interpretation in this framework, which together characterize a broad class of power indices based on dividends resulting both from exercising and from undergoing control. By adding an extra condition a unique power index is singled out. In this index, each player accumulates his Shapley-Shubik power index assignments from controlling other players, diminished by the sum of the Shapley-Shubik power index assignments to other players controlling him.
\end{abstract}

Keywords: Mutual control structure, simple games, power index. JEL classification: C71, G34

\footnotetext{
*We thank participants of seminars in Montréal, Saarbrücken, Udine, Brussels, Lund, and Enschede, whose comments have led to several improvements of the paper.

${ }^{\dagger}$ Department of Economics and Statistics, Saarland University, D-66123 Saarbrücken, Germany. Email: doka@mx.uni-saarland.de.

$\ddagger$ Department of Quantitative Economics, Maastricht University, The Netherlands. Email: h.peters@maastrichtuniversity.nl.
} 


\section{Introduction}

A mutual control structure describes a situation in which agents exercise control over each other. Typically, such situations occur in the area of corporate governance/finance, when a conglomerate of firms and investment companies control each other, specifically by possessing shares or stocks. As an example, consider the Porsche-Volkswagen case, from 2008 onwards, when the Porsche group started buying common stocks of Volkswagen AG on a grand scale. On January 5, 2009, Porsche announced to own more than $50 \%$ of the common stocks. However, the original plan to take over Volkswagen failed and the founding of an integrated corporation was announced. Figure 1 is a simplified organization chart of this corporation. ${ }^{1}$ Although Porsche SE has the majority of voting rights of Volkswagen AG, Lower Saxony has a veto power according to two laws. $^{2}$ These laws specify in particular that important decisions can be made only with $80 \%$ of the voting rights (of which Lower Saxony owns slightly more than $20 \%)^{3}$

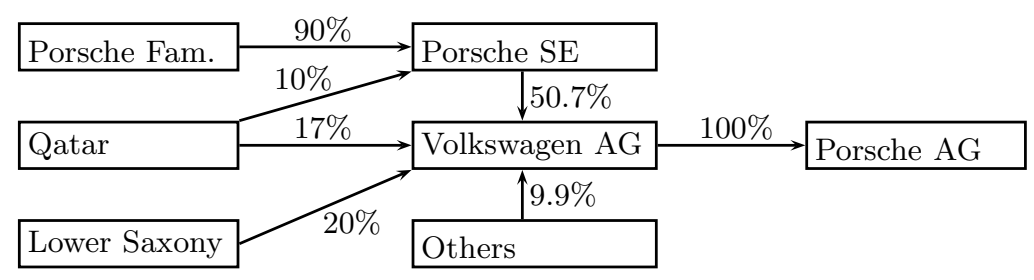

Figure 1: Porsche and VW voting rights by the end of 2012, based on the annual reports 2012 of Volkswagen AG and Porsche Automobil Holding SE GmbH.

Interesting as the historical development of this case may be, even until very recently, in this paper we will be mainly interested in the resulting organization chart as represented in Figure 1. This will serve as a recurring example (Example 2.2 in the next section, and its continuation further on). In a situation like this and in similar situations the question arises who are, ultimately, in control, and how much power the involved parties have. The purpose of the present paper is to answer these questions and contribute to the literature by developing a general game-theoretic model.

\footnotetext{
${ }^{1}$ Porsche Families is an aggregation of several companies and holdings which are held by members of the families Porsche and Piëch. Qatar is an aggregation of several holdings which are held by the Qatar Investment Authority, Doha. Lower Saxony includes Hannoversche Beteiligungsgesellschaft mbH which is held by Lower Saxony. Volkswagen AG holds $100 \%$ of Porsche Holding Stuttgart GmbH which in turn holds $100 \%$ of Porsche AG - Porsche Holding Stuttgart $\mathrm{GmbH}$ has been left out for obvious reasons. Others means investors which hold less than $3 \%$ of the shares and are therefore not mentioned in any reports.

${ }^{2}$ Namely, §111 AktG (Aktiengesetz) and $\$ 4$ VWGmbHüG (Gesetz über die Überführung der Anteilsrechte der Volkswagen Gesellschaft mit beschränkter Haftung in private Hand).

${ }^{3}$ Figure 1 describes the situation as it was before mid June, 2013. Meanwhile, Qatar has sold its shares in Porsche SE back to Porsche Families. For the sake of the example, however, we stick to the situation before mid June, 2013.
} 
Formally, a mutual control structure $C$ will be a map assigning to each nonempty coalition - i.e., a subset of a given finite set of players $N$ - another coalition. The interpretation of $C(S)=T$ is, that each player of $T$ is controlled by the coalition $S$. For instance, $i \in T$ is a firm, and the coalition $S$ of firms or investment companies has a majority of the shares of firm $i$. We impose the natural condition of monotonicity: if $S$ controls $T$, then any coalition containing $S$ also controls $T$. While the mutual control structure $C$ thus captures direct control, it does not necessarily capture indirect control. The latter means that whenever $S$ controls $T$, and $S$ and $T$ jointly control $R$, then $S$ indirectly controls $R$. Thus, if $j$ is a firm in $R$ and $S$ and $T$ jointly have a majority of the shares of $j$, then $S$ controls $j$ if it is the case that $S$ controls all firms in $T$. A mutual control structure will be called invariant if it satisfies this condition. In the paper we start out by studying a procedure which assigns to each mutual control structure its unique minimal invariant extension.

Alternatively, a mutual control structure can be described by a vector of simple games, to be called a simple game structure in the paper. For each player, there is a (monotonic) simple game with as winning coalitions exactly those coalitions which control that player. There is a one-to-one correspondence between mutual control structures and simple game structures. We propose an updating procedure, in which players can be replaced by coalitions which are winning in the simple game describing who controls that player. The steps in this procedure are called elementary substitutions. We show that by applying these elementary substitutions we obtain a unique minimal invariant extension of the simple game structure, and that the associated mutual control structure is indeed the minimal invariant extension of the originally given mutual control structure.

A relatively early approach to the problem of indirect control in the literature is Gambarelli and Owen (1994). This approach explicitly distinguishes between firms and investors. In what is called a 'reduction', all power is reduced to power of the investors, i.e., the firms leave the scene. The proposed reduction operation bears some resemblance to our procedure of making a mutual control structure invariant. Gambarelli and Owen (1994) end up with so-called consistent reductions which, however, are not necessarily unique, in contrast to our minimal invariant extensions. ${ }^{4}$

Our approach is also related to the work of $\mathrm{Hu}$ and Shapley (2003a, 2003b). If player $i$ is controlled by coalition $S$, i.e., $i \in C(S)$, then $S$ is called a 'boss set' for player $i$ in Hu and Shapley (2003a), but next to boss sets they also consider 'approval sets'. Our procedure to update mutual control structures in order to incorporate indirect control is quite similar to the one in $\mathrm{Hu}$ and Shapley (2003b), but, as mentioned, their assumptions on such a structure are different. In this respect, our approach is simpler and focusses on control ('boss sets' in

\footnotetext{
${ }^{4}$ Also Driessen and Sun (2006) distinguish between firms and investors. They consider such a situation as an application of a so-called 'set game'. The 'worth' of a coalition (of investors) is a set (of firms), meaning that the coalition of investors controls the firms in the set. In their framework a 'value' assigns to each investor a set of firms, where these sets are not necessarily disjoint.
} 
their terminology). Hu and Shapley (2003a and 2003b) also study command games, which are equivalent to our simple game structures. They propose a power index (Hu and Shapley, 2003a), which, however, seems quite different from the power indices that we arrive at, see below.

There are some other approaches in the literature aiming at establishing indirect control relations: see, for instance Crama and Leruth (2007). For a recent overview of the theoretical and empirical literature in this area see Crama and Leruth (2011).

Additionally, there is another strand of literature which considers cooperative games respecting a specific graph theoretic structure imposed on the player set, and which is to some extent related to our approach. For instance, the 'permission structure' considered in Gilles et al. (1992) can be seen as a special instance of a mutual control structure (though with a somewhat different interpretation) in which all minimal controlling coalitions are singletons.

In the second part of the paper we consider invariant mutual control structures and develop a class of power indices, intended to capture the 'true' power of individual players. We impose four axioms which have a plausible interpretation in the present framework. First, we set the power of null-players equal to zero a null-player is a player who neither contributes to controlling any other player, nor is controlled by any other player or coalition himself. Second, we impose that the sum of all assigned powers is the same over all invariant mutual control structures. This axiom replaces the usual 'efficiency' condition; it will easily follow that this axiom together with the null-player axiom actually makes this sum equal to zero. Third, we impose anonymity: the names of the players should not matter. Fourth, we impose a so-called transfer property, which says the following. For every player, the change in power when extending a mutual control structure $C^{\prime}$ to $C$ should be equal to the change in power when extending a mutual control structure $D^{\prime}$ to $D$, whenever exactly the same control relations are added going from $C^{\prime}$ to $C$ as when going from $D^{\prime}$ to $D$. This condition is called transfer property because it is related to the transfer property used by Dubey (1975) to characterize the Shapley value or Shapley-Shubik index (Shapley, 1953; Shapley and Shubik, 1954) for monotonic simple games. See also Dubey et al. (2005).

We characterize the class of power indices satisfying these four conditions. Each power index in this class corresponds to a weight vector of dimension $2 n-2$ (where $n$ is the number of players) and assigns to a player $i$ a weighted sum of dividends obtained in the simple games capturing the control undergone by the other players, diminished by dividends gathered by the other players in the game describing the control undergone by player $i$. The number $2 n-2=2(n-1)$ is twice the number of possible cardinalities of a (nontrivial) coalition: for each coalition there is a weight associated with control exercised by that coalition, and a weight associated with control undergone by that coalition. By adding a natural monotonicity condition we obtain power indices with nonnegative weight vectors. By imposing more scaling we obtain a unique power index with all weights equal to 1 . This means that each player $i$ obtains the sum of all his 
Shapley-Shubik values in the games in which he contributes to controlling the other players, minus the sum of all Shapley-Shubik values of the other players in the game describing the control undergone by $i$.

$\mathrm{Hu}$ and Shapley (2003a) also propose a power index for their command games, which, as already mentioned, are equivalent to our simple games in a simple game structure. If the number of players is $n$, then for each of the $n$ command games they take the Shapley-Shubik power index and put these together in an $n \times n$ Markov matrix. Then the overall power index, called 'authority distribution' is defined to be the stationary state of this matrix. Thus, this authority distribution can be obtained by starting with an arbitrary distribution and 'updating' it using the Shapley-Shubik power indices of the separate 'command' (i.e., control) games.

The approach in this paper can be applied whenever elementary, direct control relations can be retrieved from the data - for instance by considering simple majority share holdings within a corporate structure and/or taking into account special voting rights (as in the Porsche-Volkswagen case in the next section). Next, indirect control relations can be determined, and to the resulting invariant mutual control structure a power index can be applied. It should be noted, however, that it is probably impossible to capture all potential situations within an area of application in a completely satisfactory way. See the final section for some discussion.

As emphasized by Crama and Leruth (2011), in corporate governance/finance it is important to distinguish between ownership and control. Also this point will be discussed in the final section of the paper.

In Section 2 we introduce and study mutual control structures, in Section 3 we consider the approach by simple games, and in Section 4 we develop our class of power indices. Section 5 concludes with further discussion.

\section{Mutual control structures}

We start with some notations. For a set $A$ we denote by $P(A)$ the set of all subsets of $A$. By $|A|$ we denote the number of elements of $A$.

Let $N=\{1, \ldots, n\}$ with $n \geq 2$ denote the set of players. Elements of $P(N)$ are called coalitions.

Definition 2.1. A mutual control structure (mcs) is a map $C: P(N) \rightarrow P(N)$ satisfying

(i) $C(\emptyset)=\emptyset$,

(ii) monotonicity: $C(S) \subseteq C(T)$ for all $S, T \in P(N)$ with $S \subseteq T$.

The set of all mutual control structures is denoted by $\mathcal{C}$.

If $i \in C(S)$ for some $i \in N$ and $S \in P(N)$ then we say that player $i$ is controlled by coalition $S$. (For instance, the firms in $S$ hold a majority 
of the shares of firm $i$.) Similarly, we say that $S$ controls $C(S)$. Thus, the empty coalition controls no one, and a player controlled by a coalition $S$ is also controlled by any coalition $T$ containing $S$.

Example 2.2. Consider the Porsche-Volkswagen example in Figure 1. We assume that the group of 'others' does not vote, i.e., we ignore them in the description of the game. This is reasonable since these shareholders have only small amounts of shares and are never needed for control. ${ }^{5}$ We can describe the situation by a mutal control structure $C$. The players are Porsche Families (1), Qatar (2), Lower Saxony (3), Porsche SE (4), Volkswagen AG (5), and Porsche AG (6). For any coalition $S \subseteq N=\{1, \ldots, 6\}$ we have:

$$
4 \in C(S) \Leftrightarrow 1 \in S, 5 \in C(S) \Leftrightarrow\{2,3,4\} \subseteq S, \text { and } 6 \in C(S) \Leftrightarrow 5 \in S .
$$

Clearly, $C$ satisfies the conditions of Definition 2.1.

A mutual control structure does not necessarily capture 'indirect' control: in Example 2.2 Porsche Families controls Porsche SE which, together with Qatar and Lower Saxony, controls Volkswagen. But $C(\{1,2,3\}=\{4\}$, that is, Volkswagen is not controlled by $\{1,2,3\}$. However, this coalition controls Volkswagen 'indirectly' as it can enforce Porsche SE to control Volkswagen. This property of 'indirect control' is captured by the following definition.

Definition 2.3. The mutual control structure $C$ is invariant if it satisfies the following condition.

Indirect control: For all $S, T, R \in P(N)$ with $T \subseteq C(S)$ and $R \subseteq C(S \cup T)$ we have $R \subseteq C(S)$.

The set of all invariant mutual control structures is denoted by $\mathcal{C}^{*}$.

Clearly, the control structure $C$ in Example 2.2 violates indirect control with $S=\{1,2,3\}, T=\{4\}$, and $R=\{5\}$.

The term 'invariant' reflects the fact that such a mutual control structure does not change if we add further control relations in the sense of the indirect control property: if $T$ is controlled by $S$ and $R$ is controlled by $S$ and $T$ jointly, then $R$ is already controlled directly by $S$ and, thus, adding this control relation does not change the mcs.

Note that an invariant mutual control structure $C$ is transitive: for all $S, T, R \in P(N)$ with $T \subseteq C(S)$ and $R \subseteq C(T)$ we have $R \subseteq C(S)$. This follows since $R \subseteq C(T)$ implies $R \subseteq C(S \cup T)$ by monotonicity of $C$.

Examples of (invariant) mutual control structures can be derived from simple games. A simple game is a map $v: P(N) \rightarrow\{0,1\}$ with $v(\emptyset)=0$ and with $v(S) \leq v(T)$ for all $S, T \in P(N)$ with $S \subseteq T{ }^{6}$ If $v(S)=1$ then coalition $S$ is winning, otherwise it is losing. The set of all simple games is denoted by $\Sigma$.

\footnotetext{
${ }^{5}$ Alternatively, we could include them as a player: this player would then be a null-player as defined in Section 4.

${ }^{6}$ Thus, in our paper, a simple game - like a mutual control structure - is monotonic by definition. Also observe that $v(N)=0$ - and, thus, $v(S)=0$ for every coalition $S-$ is allowed.
} 
Example 2.4. Let $v \in \Sigma$ and define $C: P(N) \rightarrow P(N)$ by

$$
C(S)= \begin{cases}N & \text { if } v(S)=1 \\ \emptyset & \text { if } v(S)=0\end{cases}
$$

for all $S \in P(N)$. Then $C(\emptyset)=\emptyset$, and $C(S) \subseteq C(T)$ for all $S, T \in P(N)$ with $S \subseteq T$, so $C \in \mathcal{C}$. Also, if $S, T, R \in P(N)$ with $T \subseteq C(S)$ and $R \subseteq C(S \cup T)$ then either $T=\emptyset$ in which case $R \subseteq C(S)$ or $T \neq \emptyset$ in which case $C(S)=N$ and thus $R \subseteq C(S)$ as well. Hence, $C$ satisfies indirect control, so that $C \in \mathcal{C}^{*}$.

The remainder of this section is devoted to the question how an arbitrary mutual control structure (mcs) can be turned into an invariant mutual control structure (imcs). This requires, in particular, that indirect control relations are incorporated explicitly into the mcs. Let $C$ be an arbitrary mcs. We define $C^{1}, C^{2}, \ldots$ recursively by ${ }^{7}$

$$
C^{k}(S)= \begin{cases}C(S) & \text { if } k=1 \\ C\left(C^{k-1}(S) \cup S\right) & \text { if } k>1\end{cases}
$$

for each $S \in P(N)$. Observe that in each step of this algorithm the new coalition controlled by $S$ is the coalition controlled by $S$ jointly with the coalition already controlled by $S$ according to the previous step. Thus, this definition is very much in the spirit of the definition of indirect control. Clearly, by monotonicity of $C$, there must be a natural number $p \geq 1$ such that for each $S \in P(N)$ we have ${ }^{8}$

$$
C(S)=C^{1}(S) \subseteq C^{2}(S) \subseteq \ldots \subseteq C^{p}(S)=C^{p+1}(S)=C^{p+2}(S)=\ldots
$$

Denote $C^{p}$ by $C^{*}$. Then we have the following result, which says that in $C^{*}$ adding players controlled by a coalition $S$ to $S$ does not enlarge the set of players controlled by $S$.

Lemma 2.5. Let $C \in \mathcal{C}$. Then for each $S \in P(N)$ we have $C^{*}\left(S \cup C^{*}(S)\right)=$ $C^{*}(S)$.

Proof. Let $p \geq 1$ be as in the definition of $C^{*}$ (i.e., as in (1)) and let $S \in P(N)$. Then we have

$$
C\left(S \cup C^{*}(S)\right)=C\left(S \cup C^{p}(S)\right)=C^{p+1}(S)=C^{*}(S)
$$

hence

$$
C^{2}\left(S \cup C^{*}(S)\right)=C\left(S \cup C^{*}(S) \cup C\left(S \cup C^{*}(S)\right)\right)=C\left(S \cup C^{*}(S)\right)=C^{*}(S) .
$$

By repeating this argument we find $C^{*}\left(S \cup C^{*}(S)\right)=C^{p}\left(S \cup C^{*}(S)\right)=C^{*}(S)$.

A few further observations are collected in the following lemma. We omit the straightforward induction proofs. For $C, D \in \mathcal{C}$, we write $C \subseteq D$ if $C(S) \subseteq D(S)$ for all $S \in P(N)$.

\footnotetext{
${ }^{7}$ This procedure is also used in Hu and Shapley (2003b).

${ }^{8}$ It is not difficult to see that $p$ does not have to exceed the number of players $n$.
} 
Lemma 2.6. Let $C \in \mathcal{C}$. Then $C^{k} \in \mathcal{C}$ for each $k \geq 1$. If $D \in \mathcal{C}$ with $C \subseteq D$, then $C^{k} \subseteq D^{k}$ for each $k \geq 2$.

We now show that $C^{*}$ is an invariant mcs.

Proposition 2.7. Let $C \in \mathcal{C}$. Then $C^{*} \in \mathcal{C}^{*}$.

Proof. In view of Lemma 2.6 we only still have to show indirect control of $\mathcal{C}^{*}$. Let $S \in P(N)$ and $T \subseteq C^{*}(S)$. Then by monotonicity of $C^{*}$ and Lemma 2.5 we have

$$
C^{*}(S) \subseteq C^{*}(S \cup T) \subseteq C^{*}\left(S \cup C^{*}(S)\right)=C^{*}(S),
$$

so that $C^{*}(S \cup T)=C^{*}(S)$. Hence, if $R \subseteq C^{*}(S \cup T)$ then $R \subseteq C^{*}(S)$, so that indirect control holds.

We call $D \in \mathcal{C}^{*}$ an invariant extension of $C \in \mathcal{C}$ if $C \subseteq D$. Clearly, $C^{*}$ is an invariant extension of $C$, but also $D \in \mathcal{C}^{*}$ defined by $D(S)=N$ for all $S \in P(N)$ is an invariant extension of any mutual control structure. Call an invariant extension $D$ of $C$ minimal if $D(S) \subseteq D^{\prime}(S)$ for every $S \in P(N)$ and every invariant extension $D^{\prime}$ of $C$. Obviously, minimal invariant extensions are unique. We have the following result.

Proposition 2.8. Let $C \in \mathcal{C}$. Then $C^{*}$ is the (unique) minimal invariant extension of $C$.

Proof. Clearly, $C^{*}$ is an invariant extension of $C$. Let $D \in \mathcal{C}^{*}$ be an invariant extension of $C$ and let $S \in P(N)$. It is sufficient to prove that $C^{r}(S) \subseteq D(S)$ for all $r \geq 1$. For $r=1$ this follows by definition of an invariant extension. Suppose the claim is true for $r-1$. Then $C^{r}(S)=C\left(S \cup C^{r-1}(S)\right) \subseteq D\left(S \cup C^{r-1}(S)\right)$ again by definition of an invariant extension. By the induction hypothesis, $C^{r-1}(S) \subseteq D(S)$. Hence by indirect control of $D$ we obtain $C^{r}(S) \subseteq D(S)$.

The following example is the Porsche-Volkswagen case.

Example 2.9. For $C$ as in Example 2.2 we have for all $S \in P(N)$ :

$$
\begin{aligned}
& 4 \in C^{*}(S) \Leftrightarrow 1 \in S \\
& 5 \in C^{*}(S) \Leftrightarrow\{2,3,4\} \subseteq S \text { or }\{1,2,3\} \subseteq S \\
& 6 \in C^{*}(S) \Leftrightarrow 5 \in S \text { or }\{2,3,4\} \subseteq S \text { or }\{1,2,3\} \subseteq S .
\end{aligned}
$$

In particular, in $\mathcal{C}^{*}$, Volkswagen AG (5) is controlled by the coalition of Porsche Families, Qatar, and Lower Saxony (1, 2, and 3, respectively).

The next example shows that different mutual control structures may result in the same invariant mes.

Example 2.10. Let $N=\{1,2,3\}$ and let $C, D \in \mathcal{C}$ be defined by monotonicity and $C(\{1\})=\{2\}, C(\{2\})=\{3\}, C(\{3\})=\{1\}, D(\{1\})=\{3\}, D(\{2\})=\{1\}$, $D(\{3\})=\{2\}$. Then $C^{*}(S)=D^{*}(S)=N$ for each nonempty coalition $S$. 
We conclude this section with a remark on the union and intersection of invariant mutual control structures. This will be relevant in particular when we discuss axioms for power indices later on.

Remark 2.11. We define $\cup$ and $\cap$ on $\mathcal{C}$ by

$$
\begin{aligned}
& (C \cup D)(S)=C(S) \cup D(S), \\
& (C \cap D)(S)=C(S) \cap D(S),
\end{aligned}
$$

for all $S \in P(N)$. Clearly, $C \cup D, C \cap D \in \mathcal{C}$ for all $C, D \in \mathcal{C}$. Let $C, D \in \mathcal{C}^{*}$ and consider $C \cap D$. If $T \subseteq(C \cap D)(S)$ and $R \subseteq(C \cap D)(S \cup T)$, then $R \subseteq C(S)$ and $R \subseteq D(S)$ by indirect control. Hence, $R \subseteq(C \cap D)(S)$. Thus, $C \cap D \in \mathcal{C}^{*}$.

However, $C \cup D$ does not have to be invariant. Consider the following mutual control structures:

$$
C(S)=\left\{\begin{array}{cc}
\{2\} & \text { if } 1 \in S \\
\emptyset & \text { otherwise }
\end{array}\right.
$$

and

$$
D(S)=\left\{\begin{array}{cc}
\{3\} & \text { if } 2 \in S \\
\emptyset & \text { otherwise. }
\end{array}\right.
$$

Then $(C \cup D)(\{1\})=\{2\},\{2,3\}=(C \cup D)(\{1,2\})$, but $\{2,3\} \nsubseteq(C \cup D)(\{1\})$. So $C \cup D$ is not invariant.

In the next section we consider the representation of a mutual control structure by simple games.

\section{Mutual control structures and simple games}

Let $C$ be an mcs. Instead of writing for each coalition all players it controls, we may equivalently write for each player all coalitions by which he is controlled. Formally, for each $i \in N$ we define a simple game $w_{i}^{C}$ by

$$
w_{i}^{C}(S)= \begin{cases}1 & \text { if } i \in C(S) \\ 0 & \text { if } i \notin C(S) .\end{cases}
$$

This way with every $C \in \mathcal{C}$ a vector of simple games $w^{C} \in \Sigma^{N}$ is associated. We call an element of $\Sigma^{N}$ a simple game structure. ${ }^{9}$

Conversely, for a simple game structure $w \in \Sigma^{N}$ we can define an mcs $C^{w}$ by $i \in C^{w}(S): \Leftrightarrow w_{i}(S)=1$ for all $i \in N$ and $S \in P(N)$. Clearly, these definitions determine a bijection between $\mathcal{C}$ and $\Sigma^{N}$, and all statements and results for $\mathcal{C}$ can be equivalently formulated for $\Sigma^{N}$ and conversely.

For an arbitrary $w \in \Sigma^{N}$ it is not necessarily the case that $C^{w}$ is invariant, i.e., $C^{w} \in \mathcal{C}^{*}$. For each pair $i, j \in N$ with $i \neq j$ we now define a map $t_{i, j}: \Sigma^{N} \rightarrow$ $\Sigma^{N}$, such that repeated application of these maps will transform a $w \in \Sigma^{N}$ into a $w^{*} \in \Sigma^{N}$ with the property that $C^{w^{*}}$ is invariant. Formally,

$$
t_{i, j}\left(w_{k}\right)(S)=\left\{\begin{array}{cc}
w_{k}(S) & \text { if } k \neq i \\
\widetilde{w}_{i}(S) & \text { if } k=i
\end{array}\right.
$$

\footnotetext{
${ }^{9}$ These simple games are called 'command games' in Hu and Shapley (2003a,b).
} 
for each $k \in N$, where

$\widetilde{w}_{i}(S)= \begin{cases}1 & \text { if } w_{i}(S)=1 \\ 1 & \text { if } S=\left(S_{1} \backslash\{j\}\right) \cup S_{2} \text { for some } S_{1}, S_{2} \text { with } w_{i}\left(S_{1}\right)=1, w_{j}\left(S_{2}\right)=1 \\ 0 & \text { otherwise }\end{cases}$

for all $S \in P(N)$. We call $t_{i, j}$ an elementary substitution. In $t_{i, j}(w)$, the set of winning coalitions in game $w_{i}$ is extended by replacing player $j$ in every winning coalition of $w_{i}$ by any winning coalition of $w_{j}$; i.e., $j$ is replaced by a coalition that controls $j .^{10}$ This is, clearly, in the spirit of indirect control.

Example 3.1. Recall the mutual control structure $C$ from Example 2.2 (PorscheVolkswagen), where $N=\{1, \ldots, 6\}$ and the minimal winning coalitions in $w^{C}$ are given in the following table. (We leave out the zero games $w_{1}^{C}, w_{2}^{C}$, and $\left.w_{3}^{C}.\right)$

\begin{tabular}{ccc}
\hline$w_{4}^{C}$ & $w_{5}^{C}$ & $w_{6}^{C}$ \\
\hline$\{1\}$ & $\{2,3,4\}$ & $\{5\}$ \\
\hline
\end{tabular}

Applying the transformation $t_{5,4}$ yields:

\begin{tabular}{lcc}
\hline$w_{4}^{C}$ & $w_{5}^{C}$ & $w_{6}^{C}$ \\
\hline$\{1\}$ & $\{2,3,4\},\{2,3,1\}$ & $\{5\}$ \\
\hline
\end{tabular}

and, next, application of $t_{6,5}$ yields:

\begin{tabular}{lcc}
\hline$w_{4}^{C}$ & $w_{5}^{C}$ & $w_{6}^{C}$ \\
\hline$\{1\}$ & $\{2,3,4\},\{2,3,1\}$ & $\{5\},\{2,3,4\},\{2,3,1\}$ \\
\hline
\end{tabular}

The last table describes a simple game structure $w^{*}$ (with $w_{1}^{*}, w_{2}^{*}, w_{3}^{*}$ zero games) which will not further change under elementary substitutions, and we have that $C^{w^{*}}$ is equal to $C^{*}$, with $C^{*}$ as given in Example 2.9.

Elementary substitutions are closely related to the procedure constructing invariant mcs discussed in the preceding section. Clearly, since applying elementary substitutions on a simple game structure $w$ can only increase the collections of winning coalitions, after finitely many steps we must obtain some $w^{*} \in \Sigma^{N}$ which is invariant under such substitutions. We show that the associated mcs $C^{w^{*}}$ is invariant and equal to the imcs $\left(C^{w}\right)^{*}$ obtained by applying the procedure of the preceding section to $C^{w}$. We start with the following lemma.

Lemma 3.2. Let $w \in \Sigma^{N}$ and $i, j \in N$ with $i \neq j$. Then, for each $r \geq 1$, we have

(a) $\left(C^{w}\right)^{r} \subseteq\left(C^{t_{i, j}(w)}\right)^{r}$,

(b) $\left(C^{t_{i, j}(w)}\right)^{r} \subseteq\left(C^{w}\right)^{2 r}$.

\footnotetext{
${ }^{10}$ This coalition may again contain player $j$ but that case is also covered by monotonicity.
} 
Proof. Write $u=t_{i, j}(w)$. For (a), since $u(S) \geq w(S)$ for all $S \in P(N)$, we have $C^{w}(S) \subseteq C^{u}(S)$ for all $S$, and the proof is complete by Lemma 2.6.

In order to prove (b), let $S \in P(N)$. The proof is by induction.

First suppose $r=1$. Let $k \in C^{u}(S)$, i.e., $u_{k}(S)=1$. If $k \neq i$ then $w_{k}(S)=u_{k}(S)=1$, hence $k \in C^{w}(S)=\left(C^{w}\right)^{1}(S) \subseteq\left(C^{w}\right)^{2}(S)$. This argument also holds if $k=i$ and $w_{k}(S)=1$. Now suppose $k=i$ and $w_{k}(S)=0$. Then there are $S_{1}, S_{2} \in P(N)$ with $w_{k}\left(S_{1}\right)=1, w_{j}\left(S_{2}\right)=1$, and such that $S=\left(S_{1} \backslash\{j\}\right) \cup S_{2}$. Hence $w_{j}(S) \geq w_{j}\left(S_{2}\right)=1$ so that $j \in C^{w}(S)=\left(C^{w}\right)^{1}(S)$ and $w_{k}\left(S \cup\left(C^{w}\right)^{1}(S)\right) \geq w_{k}(S \cup\{j\}) \geq w_{k}\left(S_{1}\right)=1$. Hence $k \in\left(C^{w}\right)^{2}(S)$ also in this case.

Now let $r \geq 2$ and suppose (b) holds for all $k \leq r-1$. Then

$$
\begin{aligned}
\left(C^{u}\right)^{r}(S) & =C^{u}\left(S \cup\left(C^{u}\right)^{r-1}(S)\right) \\
& \subseteq\left(C^{w}\right)^{2}\left(S \cup\left(C^{u}\right)^{r-1}(S)\right) \\
& \subseteq\left(C^{w}\right)^{2}\left(S \cup\left(C^{w}\right)^{2 r-2}(S)\right) \\
& =C^{w}\left(S \cup\left(C^{w}\right)^{2 r-2}(S) \cup C^{w}\left(S \cup\left(C^{w}\right)^{2 r-2}(S)\right)\right) \\
& =C^{w}\left(S \cup\left(C^{w}\right)^{2 r-2}(S) \cup\left(C^{w}\right)^{2 r-1}(S)\right) \\
& =C^{w}\left(S \cup\left(C^{w}\right)^{2 r-1}(S)\right) \\
& =\left(C^{w}\right)^{2 r}(S),
\end{aligned}
$$

by using the induction hypothesis and Lemma 2.6.

A consequence of this lemma is that, if we first apply an elementary substitution to simple game structure, then the associated invariant mos does not change.

Corollary 3.3. Let $w \in \Sigma^{N}$ and let $i, j \in N$ with $i \neq j$. Then $\left(C^{w}\right)^{*}=$ $\left(C^{t_{i, j}(w)}\right)^{*}$.

Proof. Write $u=t_{i, j}(w)$ and take $p \in \mathbb{N}$ such that $\left(C^{w}\right)^{r}=\left(C^{w}\right)^{*}$ and $\left(C^{u}\right)^{r}=\left(C^{u}\right)^{*}$ for all $r \geq p$. Let $S \in P(N)$. Then

$$
\left(C^{w}\right)^{*}(S)=\left(C^{w}\right)^{p}(S) \subseteq\left(C^{u}\right)^{p}(S) \subseteq\left(C^{w}\right)^{2 p}(S)=\left(C^{w}\right)^{*}(S),
$$

where the two inclusions follow from Lemma 3.2. Hence $\left(C^{u}\right)^{*}(S)=\left(C^{u}\right)^{p}(S)=$ $\left(C^{w}\right)^{*}(S)$.

As already observed, repeatedly applying elementary substitutions to a $w \in$ $\Sigma^{N}$ must result in some $w^{*} \in \Sigma^{N}$ which is invariant under further elementary substitutions, i.e., $t_{i, j}\left(w^{*}\right)=w^{*}$ for all $i, j \in N$ with $i \neq j$. This is so because by an elementary substitution the set of winning coalitions in each coordinate game can only expand. Let $T(w) \subseteq \Sigma^{N}$ denote the set of all such $w^{*}$, i.e., obtainable from $w$ by elementary substitutions and invariant under further elementary substitutions. Below (Corollary 3.6) we will actually show that $T(w)$ contains a unique element for every $w \in \Sigma^{N}$.

In general, call $\bar{w} \in \Sigma^{N}$ an extension of $w \in \Sigma^{N}$ if $\bar{w}_{i}(S) \geq w_{i}(S)$ for every $i \in N$ and $S \in P(N)$. Call $w \in \Sigma^{N}$ invariant if $t_{i, j}(w)=w$ for all $i, j \in N$ 
with $i \neq j$. Call $\bar{w} \in \Sigma^{N}$ a minimal invariant extension of $w \in \Sigma^{N}$ if $\bar{w}$ is an invariant extension of $w$ and for every invariant extension $w^{\prime} \in \Sigma^{N}$ of $w$ we have $w_{i}^{\prime}(S) \geq \bar{w}_{i}(S)$ for all $i \in N$ and $S \in P(N)$. Observe that, if a minimal invariant extension of $w$ exists, then it is unique.

We first show that, if $\bar{w}$ is an invariant extension of $w$, then it is an invariant extension of $w^{*}$ for every $w^{*} \in T(w)$.

Lemma 3.4. Let $\bar{w}$ be an invariant extension of $w \in \Sigma^{N}$ and let $w^{*} \in T(w)$. Then $\bar{w}$ is an invariant extension of $w^{*}$.

Proof. Let $i, j \in N$. It is sufficient to prove that $\bar{w}$ is an extension of $t_{i, j}(w)$. Suppose not, then by definition of $t_{i, j}$ there must be an $S_{1} \in P(N)$ with $w_{i}\left(S_{1}\right)=1$, a $j \in S_{1}$, and an $S_{2} \in P(N)$ with $w_{j}\left(S_{2}\right)=1$, such that $\bar{w}_{i}\left(\left(S_{1} \backslash\{j\}\right) \cup S_{2}\right)=0$. But then, $t_{i, j}(\bar{w})_{i}\left(\left(S_{1} \backslash\{j\}\right) \cup S_{2}\right)=1$ since $\bar{w}$ is an extension of $w$, contradicting invariance of $\bar{w}$.

As announced, in what follows we will show that $T(w)$ contains a unique element, which is also the unique minimal invariant extension of $w$. The next lemma further prepares for this result.

Lemma 3.5. Let $w \in \Sigma^{N}$. Then $w$ is invariant if and only if $C^{w}=\left(C^{w}\right)^{*}$.

Proof. For the only-if part, let $t_{i, j}(w)=w$ for all $i, j \in N$ with $i \neq j$. It is sufficient to show that $\left(C^{w}\right)^{r} \subseteq C^{w}$, hence $\left(C^{w}\right)^{r}=C^{w}$, for all $r \geq 1$. We show this by induction over $r$. For $r=1$ this is clear. Now let the claim be true for $r-1$. Let $S \in P(N)$ and $k \in\left(C^{w}\right)^{r}(S)$. Then by the induction hypothesis

$$
k \in\left(C^{w}\right)^{r}(S)=C^{w}\left(S \cup\left(C^{w}\right)^{r-1}(S)\right)=C^{w}\left(S \cup C^{w}(S)\right) .
$$

Hence there is a coalition $T \subseteq C^{w}(S) \backslash S$ such that $w_{k}(S \cup T)=1$. If $T=\emptyset$ then $w_{k}(S)=1$, hence $k \in C^{w}(S)$ and we are done. So let $T \neq \emptyset$. Then $w_{\ell}(S)=1$ for all $\ell \in T$. We show that $w_{k}(S)=1$ and, hence, $k \in C^{w}(S)$, by induction over $|T|$. Let $|T|=1$ and $\{\ell\}=T$. Then

$$
\begin{aligned}
w_{k}(S) & =w_{k}(S \cup T \backslash\{\ell\})=w_{k}((S \cup T \backslash\{\ell\}) \cup S) \\
& =t_{k, \ell}(w)_{k}(S \cup T)=w_{k}(S \cup T)=1,
\end{aligned}
$$

where the third equality follows from the definition of $t_{k, \ell}$ and the fourth from invariance of $w$. Hence $k \in C^{w}(S)$. Let now $m=|T| \geq 2$ and let the claim be true for $m-1$. This implies in particular that $w_{k}(S)=w_{k}(S \cup P)$ whenever $|P|=m-1$. Then let $\ell \in T$ and write $T=T^{\prime} \cup\{\ell\}$, where $\left|T^{\prime}\right|=m-1$. Then

$$
\begin{aligned}
w_{k}(S) & =w_{k}\left(S \cup T^{\prime}\right)=w_{k}(S \cup T \backslash\{\ell\})=w_{k}((S \cup T \backslash\{\ell\}) \cup S) \\
& =t_{k, \ell}(w)_{k}(S \cup T)=w_{k}(S \cup T)=1
\end{aligned}
$$

where the first equality follows by induction, the third equality again from the definition of $t_{k, \ell}$, and the fourth from invariance of $w$. Hence, $k \in C^{w}(S)$.

For the if-part, let $\left(C^{w}\right)^{*}=C^{w}$. Assume that there are $i, j, k \in N$ with $i \neq j$ and $T \in P(N)$ such that $t_{i, j}(w)_{k}(T) \neq w_{k}(T)$. Then we must have $i=k$ 
and $t_{i, j}(w)_{k}(T)=1$ whereas $w_{k}(T)=0$. Hence, there are coalitions $T_{1}$ and $T_{2}$ with $T=\left(T_{1} \backslash\{j\}\right) \cup T_{2}$ such that $w_{k}\left(T_{1}\right)=w_{j}\left(T_{2}\right)=1, j \in T_{1}$, and $w_{k}\left(T_{1} \backslash\{j\}\right)=0$. Hence $w_{j}(T)=1$, i.e., $j \in C^{w}(T)$, and thus $w_{k}\left(T \cup C^{w}(T)\right)=$ 1, i.e., $k \in C^{w}\left(T \cup C^{w}(T)\right)=\left(C^{w}\right)^{2}(T)$. On the other hand, $w_{k}(T)=0$ implies $k \notin C^{w}(T)$, a contradiction since $C^{w}(T)=\left(C^{w}\right)^{2}(T)$, as follows from $\left(C^{w}\right)^{*}=C^{w}$.

The preceding results now imply the existence of (unique) minimal invariant extensions.

Corollary 3.6. Let $w \in \Sigma^{N}$. Then $|T(w)|=1$. If $T(w)=\left\{w^{*}\right\}$, then

(a) $C^{w^{*}}=\left(C^{w}\right)^{*}$, and

(b) $w^{*}$ is the unique minimal invariant extension of $w$.

Proof. First, let $w^{*} \in T(w)$. We prove that $w^{*}$ satisfies (a). Therefore, let $S \in$ $P(N)$. Then $C^{w^{*}}(S)=\left(C^{w^{*}}\right)^{*}(S)$ by Lemma 3.5, and $\left(C^{w^{*}}\right)^{*}(S)=\left(C^{w}\right)^{*}(S)$ by Corollary 3.3, so that $C^{w^{*}}(S)=\left(C^{w}\right)^{*}(S)$. This completes the proof of (a). Now suppose $w^{*}, w^{\prime} \in T(w)$. Then by (a), $C^{w^{*}}=C^{w^{\prime}}$, so that $w^{*}=w^{\prime}$. Hence $|T(w)|=1$, say $T(w)=\left\{w^{*}\right\}$. Let $\bar{w}$ be any invariant extension of $w$, then by Lemma $3.4, \bar{w}$ is an invariant extension of $w^{*}$. This proves (b).

Thus, applying (in arbitrary order) elementary substitutions to $w \in \Sigma^{N}$ results in the unique minimal invariant extension $w^{*}$. We write $\left(\Sigma^{N}\right)^{*}:=\{w \in$ $\Sigma^{N} \mid w$ is invariant $\}$. The following commuting diagrams summarize the main results of this section and the preceding one.

(i)

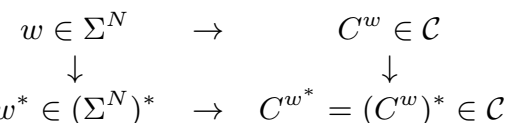
$C \in \mathcal{C} \quad \rightarrow \quad w^{C} \in \Sigma^{N}$
(ii) $\begin{aligned} & \downarrow \\ & C^{*} \\ & \in\end{aligned} \mathcal{C}^{*} \rightarrow w^{C^{*}}=\left(w^{\downarrow}\right)^{*} \in\left(\Sigma^{N}\right)^{*}$

\section{Power indices for invariant mutual control structures}

In this section we develop a class of power indices for invariant mutual control structures. Of course, such a power index can be applied to an arbitrary mutual control structure, simply by applying it to its unique invariant extension. ${ }^{11}$

As before, the player set is denoted by $N=\{1, \ldots, n\}$, and $\mathcal{C}^{*}$ is the set of all imcs with this player set. A power index is a map $\varphi: \mathcal{C}^{*} \rightarrow \mathbb{R}^{N}$.

For an imcs $C$ the marginal control of player $i \in N$ with respect to a coalition $S \subseteq N$ is defined as $\Delta_{i}^{C}(S)=C(S) \backslash C(S \backslash\{i\})$. We say that $i \in N$ is a null player (with respect to $C$ ) if $\Delta_{i}^{C}(S)=\emptyset$ and $i \notin C(S)$ for all $S \subseteq N$. That is, player $i$ is a null player if $i$ is never needed by any coalition to exercise its control, and $i$ is also not controlled by any coalition. The imcs in which every player is a null player, is denoted by $O$, i.e., $O(S)=\emptyset$ for all $S \subseteq N$.

\footnotetext{
${ }^{11}$ Attempts to define and characterize power indices directly on the class of all mutual control structures have not been fruitful.
} 
Let $\pi: N \rightarrow N$ be a permutation. Then we define $\pi C \in \mathcal{C}^{*}$ by

$$
(\pi C)(S)=\pi\left(C\left(\pi^{-1}(S)\right)\right) .
$$

The first two axioms that we impose on a power index, are as follows.

Null-Player (NP) $\varphi_{i}(C)=0$ for every null player $i$ with respect to $C$, for every $C \in \mathcal{C}^{*}$.

Constant-Sum (CS) $\sum_{i \in N} \varphi_{i}(C)=\sum_{i \in N} \varphi_{i}(D)$ for all $C, D \in \mathcal{C}^{*}$.

The null-player axiom sets the power of a player who neither controls nor is controlled, equal to 0 . The constant-sum axiom normalizes the power index over different mutual control structures, and plays the role of the usual efficiency axiom. The following observation is almost immediate.

Lemma 4.1. Let $\varphi$ be a power index satisfying NP and CS. Then $\sum_{i \in N} \varphi_{i}(C)=$ 0 for every $C \in \mathcal{C}^{*}$.

Proof. By NP, $\varphi_{i}(O)=0$ for every $i \in N$. Hence, by CS, $\sum_{i \in N} \varphi_{i}(C)=$ $\sum_{i \in N} \varphi_{i}(O)=0$ for every $C \in \mathcal{C}^{*}$.

The other two basic axioms are the following.

Anonymity (AN) $\varphi_{\pi(i)}(\pi C)=\varphi_{i}(C)$ for every player $i \in N$, every permutation $\pi$ of $N$, and every $C \in \mathcal{C}^{*}$.

Transfer Property (TP) $\varphi(C)-\varphi\left(C^{\prime}\right)=\varphi(D)-\varphi\left(D^{\prime}\right)$ for all $C, C^{\prime}, D, D^{\prime} \in$ $\mathcal{C}^{*}$ such that $C^{\prime} \subseteq C, D^{\prime} \subseteq D$, and $C(S) \backslash C^{\prime}(S)=D(S) \backslash D^{\prime}(S)$ for every $S \subseteq N$.

The anonymity axiom needs no further explanation. The transfer property says that if going from $C^{\prime}$ to $C$ involves exactly the same increase in control as going from $D^{\prime}$ to $D$, then the power of each player should change by the same amount when going from $C^{\prime}$ to $C$ as when going from $D^{\prime}$ to $D$. The transfer property is related to a property with the same name, used to characterize the Shapley value (Shapley, 1953) for (monotonic) simple games (Dubey, 1975). The form in which we present it is closely related to a version of the axiom discussed in Dubey et al. (2005) and Einy and Haimanko (2011). The following lemma shows that TP is equivalent to a condition closely related to the original format of the transfer axiom as introduced in Dubey (1975).

Lemma 4.2. Let $\varphi$ be a power index. Then $\varphi$ satisfies TP if and only if

$$
\varphi(C \cap D)+\varphi(C \cup D)=\varphi(C)+\varphi(D)
$$

for all $C, D \in \mathcal{C}^{*}$ with $C \cup D \in \mathcal{C}^{*} .{ }^{12}$

\footnotetext{
${ }^{12}$ Note that $C \cap D \in \mathcal{C}^{*}$, see Remark 2.11 .
} 
Proof. First, let $\varphi$ satisfy TP and let $C, D \in \mathcal{C}^{*}$ with $C \cup D \in \mathcal{C}^{*}$. Clearly,

$$
(C(S) \cup D(S)) \backslash C(S)=D(S) \backslash(C(S) \cap D(S))
$$

for all $S \subseteq N$. Hence by TP, $\varphi(C \cup D)-\varphi(C)=\varphi(D)-\varphi(C \cap D)$, implying (2).

Next, let $\varphi$ satisfy (2) for all $C, D \in \mathcal{C}^{*}$ with $C \cup D \in \mathcal{C}^{*}$. We show that $\varphi$ satisfies TP. Let $C, D \in \mathcal{C}^{*}$ such that $C(S) \backslash C^{\prime}(S)=D(S) \backslash D^{\prime}(S)$ for all $S \subseteq N$ and define

$$
E(S)=\bigcup_{T \subseteq S} C(T) \backslash C^{\prime}(T)=\bigcup_{T \subseteq S} D(T) \backslash D^{\prime}(T) .
$$

Clearly, $E \in \mathcal{C}$. Let $p \geq 1$ such that $E^{*}=E^{p}$. If $p=1$ then $E=E^{*} \in \mathcal{C}^{*}$. Suppose $p \geq 2$. If $i \in E^{*}(S)$ then there are coalitions $T_{1}, \ldots, T_{p-1}$ such that

$$
\begin{aligned}
& T_{1} \subseteq E(S), \\
& T_{k} \subseteq E\left(S \cup \bigcup_{l=1}^{k-1} T_{l}\right) \text { for } k=1, \ldots, p-1, \\
& i \in E\left(S \cup \bigcup_{k=1}^{p-1} T_{k}\right) .
\end{aligned}
$$

In particular, we have $T_{1} \subseteq C(S), T_{k} \subseteq C\left(S \cup \bigcup_{l=1}^{k-1} T_{l}\right)$ for $k=1, \ldots, p-1$, and $i \in C\left(S \cup \bigcup_{k=1}^{p-1} T_{k}\right)$. As $C$ is invariant, we have $i \in C(S)$, hence $E^{*}(S) \subseteq C(S)$ for all $S \subseteq N$. Hence, $C \supseteq C^{\prime} \cup E^{*}$. Also, if $j \in C(S)$ and $j \notin C^{\prime}(S)$, then $j \in E(S) \subseteq E^{*}(S)$, so that $C \subseteq C^{\prime} \cup E^{*}$. Thus, $C=C^{\prime} \cup E^{*}$. We show that

$$
C^{\prime}(S) \cap E^{*}(S)=D^{\prime}(S) \cap E^{*}(S)
$$

for all $S \subseteq N$. For this purpose let $i \in C^{\prime}(S) \cap E^{*}(S)$. Then there are $T_{1}, \ldots, T_{p-1}$ as in (3). By definition of $E$ there is $R \subseteq S \cup \bigcup_{k=1}^{p-1} T_{k}$ such that $i \in C(R) \backslash C^{\prime}(R)=D(R) \backslash D^{\prime}(R)$. In particular, $i \in D(R)$. We also have $T_{1} \subseteq E(S) \subseteq D(S)$ and $T_{k} \subseteq E\left(S \cup \bigcup_{l=1}^{k-1} T_{l}\right) \subseteq D\left(S \cup \bigcup_{l=1}^{k-1} T_{l}\right)$ so that $i \in D(S)$ by invariance of $D$. Finally, we have $i \in C^{\prime}(S)$ and therefore $i \notin C(S) \backslash C^{\prime}(S)=$ $D(S) \backslash D^{\prime}(S)$. Hence, $i \in D^{\prime}(S)$. Thus, $C^{\prime}(S) \cap E^{*}(S) \subseteq D^{\prime}(S) \cap E^{*}(S)$. The converse inclusion is analogous, so (4) holds. As $C^{\prime} \cap E^{*}$ and $D^{\prime} \cap E^{*}$ are invariant by Remark 2.11, we find with (2) that

$$
\begin{aligned}
\varphi(C)-\varphi\left(C^{\prime}\right) & =\varphi\left(C^{\prime} \cup E^{*}\right)-\varphi\left(C^{\prime}\right) \\
& =\varphi\left(E^{*}\right)-\varphi\left(C^{\prime} \cap E^{*}\right) \\
& =\varphi\left(E^{*}\right)-\varphi\left(D^{\prime} \cap E^{*}\right) \\
& =\varphi\left(D^{\prime} \cup E^{*}\right)-\varphi\left(D^{\prime}\right) \\
& =\varphi(D)-\varphi\left(D^{\prime}\right),
\end{aligned}
$$

hence, $\varphi$ satisfies TP.

Our main theorem will be a characterization of all power indices satisfying NP, CS, AN, and TP. A crucial result for this is the proposition below, which 
expresses the power index values assigned to an imcs $C$ as sums of values assigned to so-called unanimity mcs. For every $S \subseteq N$ and $j \in N$ we define the unanimity mutual control structure $U^{S,\{j\}}$ by

$$
U^{S,\{j\}}(T)=\left\{\begin{array}{cl}
\{j\} & \text { if } S \subseteq T \\
\emptyset & \text { otherwise. }
\end{array}\right.
$$

It is easy to check that $U^{S,\{j\}}$ is an imcs. Similarly, it is easy to check that $\bigcap_{S \in M} U^{S,\{j\}} \in \mathcal{C}^{*}$ for any subset $M \subseteq P(N)$ (see also Remark 2.11).

Let $C \in \mathcal{C}^{*}$. For a coalition $S \subseteq N$ we define the excess of $S$ by

$$
E^{C}(S)=C(S) \backslash \bigcup_{S^{\prime} \subsetneq S} C\left(S^{\prime}\right) .
$$

Coalition $S$ is minimal controlling in $C$ if $E^{C}(S) \neq \emptyset$. Note that $i \in E^{C}(S)$ if and only if $S$ is a minimal winning coalition in $w_{i}^{C}$ (i.e., $S$ is winning and all proper subsets of $S$ are losing). Let $M(C)$ denote the set of minimal controlling coalitions in $C$.

Example 4.3. Consider the invariant mutual control structure from Examples 2.9 and 3.1 (Porsche-Volkswagen). The minimal controlling coalitions are $\{1\}$, $\{5\},\{1,2,3\}$, and $\{2,3,4\}$. Note that $C^{*}(\{1,5\})=\{4,6\}$, so that both $C^{*}(\{1\})$ and $C^{*}(\{5\})$ are proper subsets of $C^{*}(\{1,5\})$. Still, $\{1,5\}$ is not minimal controlling since $E^{C^{*}}(\{1,5\})=\emptyset$.

We now come to the announced proposition.

Proposition 4.4. Let $\varphi$ be a power index satisfying satisfy TP and NP, and let $C \in \mathcal{C}^{*}$. For every $j \in N$ let $M^{j}=\left\{S \in M(C) \mid j \in E^{C}(S)\right\}$. Then

$$
\varphi(C)=\sum_{j \in N} \sum_{t=1}^{\left|M^{j}\right|}(-1)^{t+1} \sum_{M \subseteq M^{j},|M|=t} \varphi\left(\bigcap_{S \in M} U^{S,\{j\}}\right) .
$$

Proof. Let $C \in \mathcal{C}^{*}$. Then one easily checks that

$$
C=\bigcup_{S \in M(C)} \bigcup_{j \in E^{C}(S)} U^{S,\{j\}}=\bigcup_{j \in N} \bigcup_{S \in M^{j}} U^{S,\{j\}} .
$$

For each $j \in N$ we define the control structures $C^{j}$ and $C^{-j}$ by

$$
\begin{aligned}
C^{j} & =\bigcup_{S \in M^{j}} U^{S,\{j\}} \\
C^{-j} & =\bigcup_{k \in N \backslash\{j\}} C^{k} .
\end{aligned}
$$

It is easy to see that $C^{j}$ is an invariant mcs. Also $C^{-j}$ is an mcs, and we show that it is invariant, as follows. Let $T \subseteq N$, and suppose $\ell \in N$ with 
$\ell \notin C^{-j}(T)$. It is sufficient to show that $\ell \notin C^{-j}\left(T \cup C^{-j}(T)\right)$. If $\ell=j$, then clearly $\ell \notin C^{-j}\left(T \cup C^{-j}(T)\right)$. Now suppose $\ell \neq j$. Since $\ell \notin C^{j}(T)$ and $\ell \notin C^{-j}(T)$, we have $\ell \notin C^{j}(T) \cup C^{-j}(T)=C(T)$. Since $C$ is invariant, this implies $\ell \notin C(T \cup C(T))$. Now

$$
\begin{aligned}
C^{-j}\left(T \cup C^{-j}(T)\right) & \subseteq C^{-j}\left(T \cup C^{-j}(T) \cup C^{j}(T)\right) \\
& \subseteq C^{-j}(T \cup C(T)) \cup C^{j}(T \cup C(T)) \\
& =C(T \cup C(T))
\end{aligned}
$$

so that $\ell \notin C^{-j}\left(T \cup C^{-j}(T)\right)$.

By TP and Lemma 4.2 we have

$$
\varphi(C)=\varphi\left(C^{-1}\right)+\varphi\left(C^{1}\right)-\varphi\left(C^{-1} \cap C^{1}\right)=\varphi\left(C^{-1}\right)+\varphi\left(C^{1}\right)
$$

where the last equality follows by NP, noting that $C^{-1} \cap C^{1}=O$. Repeating this argument for $C^{-1}, C^{-2}, \ldots$, results in

$$
\varphi(C)=\sum_{j \in N} \varphi\left(C^{j}\right) .
$$

Let $j \in N$. It remains to show that

$$
\varphi\left(C^{j}\right)=\sum_{t=1}^{\left|M^{j}\right|}(-1)^{t+1} \sum_{M \subseteq M^{j},|M|=t} \varphi\left(\bigcap_{S \in M} U^{S,\{j\}}\right) .
$$

For $\left|M^{j}\right|=1$ there is nothing to show. For $\left|M^{j}\right|=2$, say $M^{j}=\left\{S_{1}, S_{2}\right\}$, we have by TP and Lemma 4.2 that

$$
\begin{aligned}
\varphi\left(C^{j}\right) & =\varphi\left(U^{S_{1},\{j\}} \cup U^{S_{2},\{j\}}\right) \\
& =\varphi\left(U^{S_{1},\{j\}}\right)+\varphi\left(U^{S_{2},\{j\}}\right)-\varphi\left(U^{S_{1},\{j\}} \cap U^{S_{2},\{j\}}\right)
\end{aligned}
$$

which results in the desired expression. So suppose $\left|M^{j}\right| \geq 3$ and let $S^{*} \in M^{j}$. By induction we have

$$
\begin{aligned}
& \varphi\left(\bigcup_{S \in M^{j} \backslash\left\{S^{*}\right\}} U^{S,\{j\}} \cap U^{S^{*},\{j\}}\right)=\varphi\left(\bigcup_{S \in M^{j} \backslash\left\{S^{*}\right\}} U^{S \cup S^{*},\{j\}}\right) \\
& =\sum_{t=1}^{\left|M^{j}\right|-1}(-1)^{t+1} \sum_{M \subseteq M^{j} \backslash\left\{S^{*}\right\},|M|=t} \varphi\left(\bigcap_{S \in M} U^{S \cup S^{*},\{j\}}\right) \\
& =\sum_{t=1}^{\left|M^{j}\right|-1}(-1)^{t+1} \sum_{M \subseteq M^{j} \backslash\left\{S^{*}\right\},|M|=t} \varphi\left(\bigcap_{S \in M \cup\left\{S^{*}\right\}} U^{S,\{j\}}\right) .
\end{aligned}
$$


Now

$$
\begin{aligned}
\varphi\left(C^{j}\right)= & \varphi\left(\bigcup_{S \in M^{j} \backslash\left\{S^{*}\right\}} U^{S,\{j\}}\right)+\varphi\left(U^{S^{*},\{j\}}\right) \\
-\varphi\left(\bigcup_{S \in M^{j} \backslash\left\{S^{*}\right\}} U^{S,\{j\}} \cap U^{S^{*},\{j\}}\right) & \\
= & \sum_{t=1}^{\left|M^{j}\right|-1}(-1)^{t+1} \sum_{M \subseteq M^{j} \backslash\left\{S^{*}\right\},|M|=t} \varphi\left(\bigcap_{S \in M} U^{S,\{j\}}\right)+\varphi\left(U^{S^{*},\{j\}}\right) \\
& -\sum_{t=1}^{\left|M^{j}\right|-1}(-1)^{t+1} \sum_{M \subseteq M^{j} \backslash\left\{S^{*}\right\},|M|=t} \varphi\left(\bigcap_{S \in M \cup\left\{S^{*}\right\}} U^{S,\{j\}}\right) \\
= & \sum_{t=1}^{\left|M^{j}\right|}(-1)^{t+1} \sum_{M \subseteq M^{j},|M|=t} \varphi\left(\bigcap_{S \in M} U^{S,\{j\}}\right)
\end{aligned}
$$

where the first and third equalities follow by TP and Lemma 4.2, and the second by (6) and induction. This completes the proof of the lemma.

Recall that the Shapley value (Shapley, 1953) of a simple game $w_{i}$ with player set $N$ is defined by

$$
S h_{k}\left(w_{i}\right)=\sum_{S \subseteq N \backslash\{k\}} \frac{|S| !(|N|-|S|-1) !}{|N| !}\left(w_{i}(S \cup\{k\})-w_{i}(S)\right)
$$

for every $k \in N$. This restriction of the Shapley value to simple games is also called the Shapley-Shubik index (Shapley and Shubik, 1954). Alternatively, as is well-known, the dividends $d_{i}(S)$ of a game $w_{i}$ can be defined, recursively, by

$$
d_{i}(S)= \begin{cases}0 & \text { if } S=\emptyset \\ w_{i}(S)-\sum_{T \varsubsetneqq S} d_{i}(T) & \text { otherwise }\end{cases}
$$

for all $S \subseteq N$. Then

$$
S h_{k}\left(w_{i}\right)=\sum_{S: k \in S} \frac{d_{i}(S)}{|S|}
$$

for every $k \in N$. For an mcs $C$ and $i \in N$, we write $d_{i}^{C}$ for the dividends of $w_{i}^{C}$.

For every weight vector $\omega=\left(\alpha_{1}, \ldots, \alpha_{n-1}, \beta_{2}, \ldots, \beta_{n}\right) \in \mathbb{R}^{2 n-2}$, we define the power index $\Phi^{\omega}$ by

$$
\begin{aligned}
\Phi_{i}^{\omega}(C)= & \sum_{k \in N \backslash\{i\}}\left(\sum_{S: i \in S, k \notin S} \frac{d_{k}^{C}(S)}{|S|} \alpha_{|S|}+\sum_{S: i \in S, k \in S} \frac{d_{k}^{C}(S)}{|S|} \beta_{|S|}\right) \\
& -\sum_{k \in N \backslash\{i\}}\left(\sum_{S: i \notin S, k \in S} \frac{d_{i}^{C}(S)}{|S|} \alpha_{|S|}+\sum_{S: i \in S, k \in S} \frac{d_{i}^{C}(S)}{|S|} \beta_{|S|}\right)
\end{aligned}
$$


for all $C \in \mathcal{C}^{*}$ and $i \in N$. The expression in brackets in the first line of (7) says that player $i$ receives a kind of weighted Shapley value in the game $w_{k}^{C}$; this expresses the power player $i$ derives from his role in controlling player $k$. The weights depend, both on the size of the coalition of whose dividend player $i$ receives a share, and on whether or not the controlled player $k$ is a member of that coalition. Thus, the first line in (7) represents the total power player $i$ acquires from his role in controlling the other players. In the second line, the total (similarly weighted) power that all other players acquire from controlling player $i$, is subtracted.

The central result of this section is the following theorem, in which the class of power indices of the form $\Phi^{\omega}$ is characterized.

Theorem 4.5. Let $\varphi$ be a power index. Then $\varphi$ satisfies NP, CS, AN, and TP if and only if there is a weight vector $\omega=\left(\alpha_{1}, \ldots, \alpha_{n-1}, \beta_{2}, \ldots, \beta_{n}\right) \in \mathbb{R}^{2 n-2}$ such that $\varphi(C)=\Phi^{\omega}(C)$ for every $C \in \mathcal{C}^{*}$.

Proof. First, let $\omega=\left(\alpha_{1}, \ldots, \alpha_{n-1}, \beta_{2}, \ldots, \beta_{n}\right) \in \mathbb{R}^{2 n-2}$. We show that $\Phi^{\omega}$ satisfies the four axioms. For AN, this is obvious. When $i$ is a null-player in $C \in \mathcal{C}^{*}$, then $w_{i}^{C}(S)=0$ for every $S$, so $d_{i}^{C}(S)=0$ for every $S$. Moreover, by using the definition of a dividend it is easy to show that $d_{k}^{C}(S)=0$ for every $S$ with $i \in S$. Hence, $\Phi_{i}^{\omega}(C)=0$, which shows NP. CS, more specifically, $\sum_{i \in N} \Phi_{i}^{\omega}(C)=0$ for every $C \in \mathcal{C}^{*}$, follows almost directly from the definition of $\Phi^{\omega}$. Finally, for TP, let $C^{\prime} \subseteq C$ and $D^{\prime} \subseteq D$ satisfy $C(S) \backslash C^{\prime}(S)=D(S) \backslash D^{\prime}(S)$ for every $S$. Then it is easily verified that for every player $j$ and every coalition $S$, we have $d_{j}^{C}(S)-d_{j}^{C^{\prime}}(S)=d_{j}^{D}(S)-d_{j}^{D^{\prime}}(S)$. Hence,

$\Phi^{\omega}(C)-\Phi^{\omega}\left(C^{\prime}\right)=$

$$
\begin{aligned}
& \sum_{k \in N \backslash\{i\}}\left(\sum_{S: i \in S, k \notin S} \frac{d_{k}^{C}(S)-d_{k}^{C^{\prime}}(S)}{|S|} \alpha_{|S|}+\sum_{S: i \in S, k \in S} \frac{d_{k}^{C}(S)-d_{k}^{C^{\prime}}(S)}{|S|} \beta_{|S|}\right) \\
- & \sum_{k \in N \backslash\{i\}}\left(\sum_{S: i \notin S, k \in S} \frac{d_{i}^{C}(S)-d_{i}^{C^{\prime}} k(S)}{|S|} \alpha_{|S|}+\sum_{S: i \in S, k \in S} \frac{d_{i}^{C}(S)-d_{i}^{C^{\prime}}(S)}{|S|} \beta_{|S|}\right) \\
= & \sum_{k \in N \backslash\{i\}}\left(\sum_{S: i \in S, k \notin S} \frac{d_{k}^{D}(S)-d_{k}^{D^{\prime}}(S)}{|S|} \alpha_{|S|}+\sum_{S: i \in S, k \in S} \frac{d_{k}^{D}(S)-d_{k}^{D^{\prime}}(S)}{|S|} \beta_{|S|}\right) \\
- & \sum_{k \in N \backslash\{i\}}\left(\sum_{S: i \notin S, k \in S} \frac{d_{i}^{D}(S)-d_{i}^{D^{\prime}} k(S)}{|S|} \alpha_{|S|}+\sum_{S: i \in S, k \in S} \frac{d_{i}^{D}(S)-d_{i}^{D^{\prime}}(S)}{|S|} \beta_{|S|}\right) \\
= & \Phi^{\omega}(D)-\Phi^{\omega}\left(D^{\prime}\right)
\end{aligned}
$$

which shows TP.

For the converse, let $\varphi$ be a power index satisfying the four axioms. By NP, CS, AN, and Lemma 4.1, there are numbers $\alpha_{|S|}$ for $|S|=1, \ldots, n-1$ and $\beta_{|S|}$ 
for $|S|=2, \ldots, n$ such that for every $\emptyset \neq S \subseteq N$ and $j \in N$ we have

$$
\varphi_{i}\left(U^{S,\{j\}}\right)=\left\{\begin{array}{cl}
0 & \text { if } i \notin S \text { and } i \neq j \\
-\alpha_{|S|} & \text { if } i=j \text { and } j \notin S \\
\frac{1}{|S|} \alpha_{|S|} & \text { if } i \in S \text { and } j \notin S \\
-\frac{|S|-1}{|S|} \beta_{|S|} & \text { if } i=j \text { and } j \in S \\
\frac{1}{|S|} \beta_{|S|} & \text { if } i \in S \backslash\{j\} \text { and } j \in S .
\end{array}\right.
$$

By Proposition 4.4 and the first part of the proof it is sufficient to prove that with $\omega=\left(\alpha_{1}, \ldots, \alpha_{n-1}, \beta_{2}, \ldots, \beta_{n}\right) \in \mathbb{R}^{2 n-2},(8)$ holds for $\Phi^{\omega}$ as well. Let $w$ be the simple game structure associated with the unanimity control structure $U^{S,\{j\}}$. Then $d_{i}(T)=0$ for every $i \neq j$ and every $T \subseteq N$, whereas $d_{j}(T)=1$ for $T=S$ and $d_{j}(T)=0$ otherwise. It is now straightforward to verify (8) for $\Phi^{\omega}$.

The family of power indices in Theorem 4.5 is quite large, since the axioms put no restrictions whatsoever on the values of the weights in $\omega$. The following natural condition on a power index $\varphi$ results in nonnegativity of the weights.

Monotonicity (MO) $\varphi_{i}(C) \geq \varphi_{i}(D)$ for all $C, D \in \mathcal{C}^{*}$ and $i \in N$ such that (i) $i \in C(S) \Rightarrow i \in D(S)$ and (ii) $\Delta_{i}^{D}(S) \subseteq \Delta_{i}^{C}(S)$ for all $S \subseteq N$.

Monotonicity says that if a player (i) is at least as much controlled in $D$ as he is in $C$, and (ii) his marginal control with respect to each coalition $S$ is in $C$ at least as large as in $D$, then this player should be assigned at least as much power in $C$ than in $D$.

Corollary 4.6. Let $\varphi$ be a power index. Then $\varphi$ satisfies NP, CS, AN, TP, and $M O$ if and only if there is a weight vector $\omega=\left(\alpha_{1}, \ldots, \alpha_{n-1}, \beta_{2}, \ldots, \beta_{n}\right) \in$ $\mathbb{R}_{+}^{2 n-2}$ such that $\varphi(C)=\Phi^{\omega}(C)$ for every $C \in \mathcal{C}^{*}$.

Proof. We leave it to the reader to verify that $\Phi^{\omega}$ satisfies MO if $\omega \in \mathbb{R}_{+}^{2 n-2}$. Conversely, let $\omega=\left(\alpha_{1}, \ldots, \alpha_{n-1}, \beta_{2}, \ldots, \beta_{n}\right) \in \mathbb{R}^{2 n-2}$ and suppose $\Phi^{\omega}$ is monotonic. If $S \subseteq N, i \in S$ and $j \notin S$, then by MO we have $\Phi_{i}^{\omega}\left(U^{S,\{j\}}\right) \geq \Phi_{i}^{\omega}(O)$, hence $\alpha_{|S|} /|S| \geq 0$. If $S \subseteq N, i \in S \backslash\{j\}$ and $j \in S$, then again by MO we have $\Phi_{i}^{\omega}\left(U^{S,\{j\}}\right) \geq \Phi_{i}^{\omega}(O)$, hence $\beta_{|S|} /|S| \geq 0$. Clearly, for $|S|=1, \beta_{|S|}=0$.

A complete determination of the weights is obtained by replacing MO by the following condition.

Controlled player (CP) For all $C \in \mathcal{C}^{*}, j \in C(N)$, and $i \in N \backslash C(N)$,

$$
\varphi_{j}(C)=\left\{\begin{array}{cl}
-1 & \text { if } \Delta_{j}^{C}(S)=\emptyset \text { for all } S \subseteq N \\
\varphi_{i}(C)-1 & \text { if } \Delta_{i}^{C}(S)=\Delta_{j}^{C}(S) \text { for all } S \subseteq N .
\end{array}\right.
$$


The controlled player condition says that if $j$ is a 'controlled player', i.e., controlled by at least one coalition and, thus, by $N$, but does not himself exercise any control, then the power of $j$ is fixed at -1 . Further, if $i$ is an uncontrolled player, i.e., controlled by no coalition at all, but $i$ and $j$ exercise the same marginal control with respect to any coalition, then their difference in power is fixed at 1 , that is, $i$ gets assigned 1 more than $j$. Note that, if $\varphi$ also satisfies NP and $i$ is a null player, then the second consequence in CP implies the first. We now have the following corollary. Its proof follows again easily from examining the unanimity mutual control structures of the form $U^{S,\{j\}}$, and is left to the reader.

Corollary 4.7. There is a unique power index satisfying NP, CS, AN, TP, and $C P$, namely the power index $\Phi^{\omega}$ with $\omega=(1, \ldots, 1) \in \mathbb{R}^{2 n-2}$.

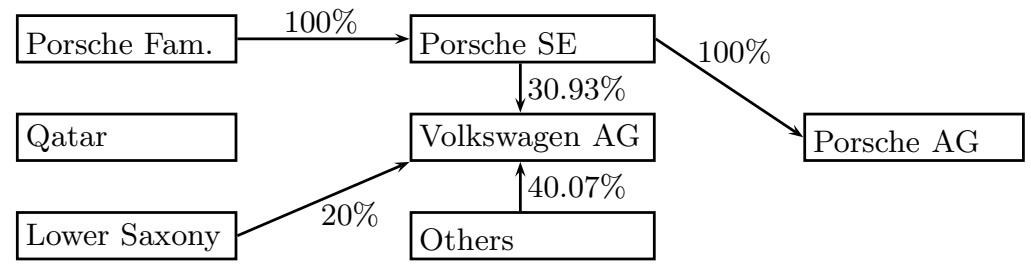

Figure 2: Porsche and VW voting rights by the end of 2007, based on the 2007 annual report of Volkswagen AG and the 2007/2008 annual report of Porsche Automobil Holding SE GmbH.

We apply this unique power index to the Porsche-Volkswagen case.

Example 4.8. Consider the invariant mutual control structure $C^{*}$ from Example 2.9. Then we find for $\omega=(1, \ldots, 1) \in \mathbb{R}^{2 n-2}$ that $\Phi_{1}^{\omega}\left(C^{*}\right)=\frac{14}{12}$, $\Phi_{2}^{\omega}\left(C^{*}\right)=\Phi_{3}^{\omega}\left(C^{*}\right)=\frac{7}{12}, \Phi_{4}^{\omega}\left(C^{*}\right)=-\frac{5}{6}, \Phi_{5}^{\omega}\left(C^{*}\right)=-\frac{1}{2}$, and $\Phi_{6}^{\omega}\left(C^{*}\right)=-1$. It is interesting to compare the power of Porsche Families with its power at the end of 2007. Figure 2 depicts the control structure between the same companies at the end of 2007. At that time, Volkswagen was not controlled by any group of main investors. Although Porsche SE has veto power in the game on Volkswagen AG, we ignore this fact, as it is not clear how this power can be exercised. We describe this situation by a mutual control structure $D$. Its minimal invariant extension is:

$$
D^{*}(S)=\{4,6\} \quad \text { for all } S \text { with } 1 \in S .
$$

Thus, even while ignoring the power of Porsche Families on Volkswagen, we still have $\Phi_{1}^{\omega}\left(D^{*}\right)=2>\frac{14}{12}=\Phi_{1}^{\omega}\left(C^{*}\right)$. Hence, according to this power index it had more power in 2007 than it has now.

We conclude this section with, first, showing the logical independence of the axioms in the main theorem and, second, discussing a variation with a variable number of players. 


\subsection{Independence of the axioms in Theorem 4.5}

We conclude with showing independence of the axioms in Theorem 4.5.

Null-Player Fix $\varepsilon>0$ and for every nonempty $S \subseteq N$ and $j \in N$ define

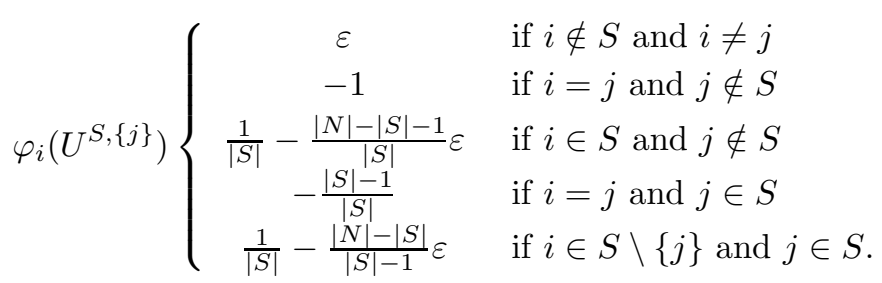

Extend $\varphi$ to $\mathcal{C}^{*}$ by using Proposition 4.4. Then $\varphi$ satisfies CS, AN, and TP, but not NP.

Constant-Sum Define the power index $\varphi$ by $\varphi_{i}(C)=|C(\{i\})|-w_{i}^{C}(N)$ for all $C \in \mathcal{C}^{*}$ and $i \in N$. Then $\varphi$ satisfies NP and AN. Also, for $C, D \in \mathcal{C}^{*}$,

$$
\begin{aligned}
\phi_{i}(C \cup D)+\phi_{i}(C \cap D)= & |(C(\{i\}) \cup D(\{i\}))|-w_{i}^{C \cup D}(N) \\
& \quad+|(C(\{i\}) \cap D(\{i\}))|-w_{i}^{C \cap D}(N) \\
= & |C(\{i\})|+|D(\{i\})|-\left(w_{i}^{C}(N)+w_{i}^{D}(N)\right) \\
= & \phi_{i}(C)+\phi_{i}(D)
\end{aligned}
$$

for all $i \in N$, so that $\varphi$ satisfies TP by Lemma 4.2. Consider $D \in \mathcal{C}^{*}$ defined by $D(S)=N$ for all nonempty $S \subseteq N$. Then $\varphi_{i}(D)=|N|-1$ for all $i \in N$, so that $\varphi$ does not satisfy CS.

Anonymity Let $N=\{1,2\}$ and define $\varphi$ by $\varphi\left(U^{\{1\},\{1\}}\right)=\varphi\left(U^{\{2\},\{2\}}\right)=$ $\varphi\left(U^{\{2\},\{1\}}\right)=(0,0), \varphi\left(U^{\{1,2\},\{2\}}\right)=-\varphi\left(U^{\{1,2\},\{1\}}\right)=\varphi\left(U^{\{1\},\{2\}}\right)=(1,-1)$; and by extending $\varphi$ to $\mathcal{C}^{*}$ using Proposition 4.4. Then $\varphi$ satisfies NP, CS, and $\mathrm{TP}$, but not AN.

Transfer property For a simple game $v$ and $i \in N$ let $\sigma_{i}(v)=\mid\{S \subseteq N \backslash\{i\} \mid$ $v(S \cup\{i\})-v(S)=1\} \mid$ and let

$$
B z_{i}(v)=\left\{\begin{array}{cl}
\sigma_{i}(v) / \sum_{j \in N} \sigma_{j}(v) & \text { if } \sigma_{i}(v) \neq 0 \\
0 & \text { if } \sigma_{i}(v)=0 .
\end{array}\right.
$$

Thus, $B z$ is the normalized Banzhaf value (Banzhaf, 1965; Dubey et al., 2005). Define the power index $\varphi$ by

$$
\varphi_{i}(C)=\sum_{j \neq i} B z_{i}\left(w_{j}^{C}\right)-\sum_{j \neq i} B z_{j}\left(w_{i}^{C}\right)
$$

for all $C \in \mathcal{C}^{*}$ and $i \in N$. It is straightforward to verify that $\varphi$ satisfies NP, $\mathrm{CS}$, and AN. We show that it does not satisfy TP by using Lemma 4.2. For 
$S, S^{\prime} \subseteq N$ define $U^{S, S^{\prime}} \in \mathcal{C}^{*}$ by $U^{S, S^{\prime}}(T)=S^{\prime}$ if $S \subseteq T$ and $U^{S, S^{\prime}}(T)=\emptyset$ otherwise. Now take $N=\{1,2,3\}$ and let $C=U^{\{1,2\}, N}$ and $D=U^{\{1,3\}, N}$. Then $B z_{1}\left(w_{i}^{C}\right)=\frac{1}{2}$ and $B z_{1}\left(w_{i}^{D}\right)=\frac{1}{2}$ for $i=1,2,3$. Hence, $\varphi_{1}(C)=\frac{1}{2}$. Further,

$$
\begin{aligned}
& C \cup D=U^{\{1,2\}, N} \cup U^{\{1,3\}, N}, \\
& C \cap D=U^{N, N} .
\end{aligned}
$$

Now $B z_{1}\left(w_{i}^{C \cup D}\right)=\frac{3}{5}$ for $i=1,2,3$. Hence, $\varphi_{1}(C \cup D)=\frac{4}{5}$. Further, by computation or by $\mathrm{CS}$ and $\mathrm{AN}$, we have $\varphi(C \cap D)=0$. Thus,

$$
\varphi_{1}(C \cup D)+\varphi_{1}(C \cap D)=\frac{4}{5} \neq 1=\varphi_{1}(C)+\varphi_{1}(D) .
$$

So, $\varphi$ does not satisfy TP.

\subsection{Variable number of players}

In view of the area of application, it is not unnatural to assume that the number of players can vary. New firms or investment companies may enter the scene, while existing players may disappear. In the Porsche-Volkswagen case, we may choose to regard the small shareholders as a null-player, or simply disregard them, as we did above. In the same case, as noted before, at mid June 2013 Qatar left the scene.

To accommodate this, let a power index $\varphi$ now be defined for every invariant mutual control structure $(N, C)$, where $N$ is an arbitrary but finite set of players, say $N \subseteq \mathbb{N}$ with $|N|<\infty$. We concentrate on a modification of Corollary 4.7 about the power index with all weights equal to one, but the other characterization results above can be modified in a similar fashion.

The axioms CS, AN, and TP are basically the same as before; we now call them $\mathrm{CS}^{*}, \mathrm{AN}^{*}$, and $\mathrm{TP}^{*}$. We modify $\mathrm{NP}$ and $\mathrm{CP}$ to $\mathrm{NP}^{*}$ and $\mathrm{CP}^{*}$, as follows.

Null-Player* $\left(\mathbf{N P}^{*}\right) \varphi_{i}(N, C)=0$ and $\varphi_{j}\left(N \backslash\{i\}, C^{\prime}\right)=\varphi_{j}(N, C)$ for every null player $i$ in $(N, C)$ and every player $j \in N \backslash\{i\}$, where $C^{\prime}(S)=C(S)$ for every $S \subseteq N \backslash\{i\}$, for every invariant mutual control structure $(N, C)$.

Controlled Player* (CP*) For every invariant mutual control structure $(N, C)$, every $j \in C(N)$, and every $i \in N \backslash C(N)$, if $\Delta_{i}^{C}(S)=\Delta_{j}^{C}(S)$ for all $S \subseteq N$, then $\varphi_{j}(C)=\varphi_{i}(C)-1$.

The modified null-player axiom $\mathrm{NP}^{*}$ not only says that null-players have zero power, but also that null-players can be left out (or added, for that matter) without affecting the power of the other players. Note that this axiom establishes a link between the values of the power index for mutual control structures with different player sets.

Compared to $\mathrm{CP}$, the $\mathrm{CP}^{*}$ version is simpler: the omitted part of $\mathrm{CP}$ can be obtained by adding a null-player. The following result can be derived almost immediately from Corollary 4.7 - we omit the proof. 
Corollary 4.9. Let the number of players be variable. There is a unique power index $\Phi^{*}$ satisfying $N P^{*}, C S^{*}, A N^{*}, T P^{*}$, and $C P^{*}$. For every invariant mutual control structure $(N, C)$, we have $\Phi^{*}(N, C)=\Phi^{\omega}(N, C)$, where $\omega=(1, \ldots, 1) \in$ $\mathbb{R}^{2|N|-2}$.

\section{Concluding remarks}

Crama and Leruth (2011) point out that the concepts of control - as studied in our paper - and ownership must be carefully distinguished. The following example illustrates this.

Example 5.1. Let $a, b, c, d$ be firms such that $a$ owns $80 \%$ of the shares of $b$ and $c$ owns the remaining $20 \%$ of $b$. Let further $a$ 's shares be distributed such that $c$ owns $40 \%$ and $d$ owns $60 \%$. See the following diagram.

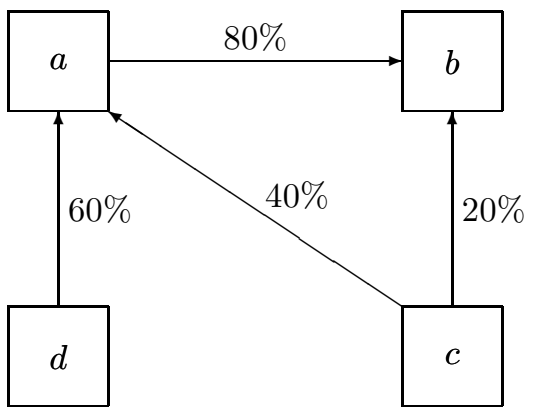

Assume that all decisions can be made by an absolute majority. From a financial point of view $c$ owns $32 \%$ of $b$ indirectly via $a$ and $20 \%$ directly, so all together $52 \%$, which is the majority. On the other hand $d$ owns $48 \%$ of $b$ indirectly via $a$. However, if we build a mutual control structure $C$ to represent this situation, we find $C(\{a\})=\{b\}, C(\{d\})=\{a\}$ and $C(\{c\})=\emptyset$. For the minimal invariant extension of $C$ we have $C^{*}(\{d\})=\{a, b\}$ and $C^{*}(\{c\})=\emptyset$. We see that indirect ownership of firms may not lead to any control at all. In particular, while indirect ownership is proportional to the number of shares, indirect control follows a winner takes all principle: although firm $c$ owns $52 \%$ of firm $b$ its indirect votes are useless as it can not force firm $a$ to use it.

As we pointed out in the introduction, our theory can be used to evaluate control structures, provided we can model them as a mutual control structure (i.e., an element of $\mathcal{C}$ ). This modelling part is important and should be done with care, as the following example illustrates.

Example 5.2. Consider five players, $N=\{1, \ldots, 5\}$, and a mutual control structure $C$ determined by $C(\{1\})=\{2\}$ and $C(\{2\})=\{3,4,5\}$. Then $C^{*}$ is determined by $C^{*}(\{1\})=\{2,3,4,5\}$ and $C^{*}(\{2\})=\{3,4,5\}$. One might argue here that player 2 should actually be left out. Indeed, if this player is merely an administrative entity (a mailbox, or an office), then it would be natural to leave 
it out and consider the mutual control structure $C^{\prime}$ with $C^{\prime}(\{1\})=\{3,4,5\}$ instead of $C$, and thus with player 2 as a null-player. If, however, player 2 is a real player, then it should be left in. In that case, one might argue that a mutual control structure $C^{\prime \prime}$ with $C^{\prime \prime}(\{1\})=\{2,3,4,5\}$ and $C^{\prime \prime}(\{2\})=\emptyset$ would reflect the situation more adequately than $C^{*}$, but this is, indeed, subject to discussion: player 2 would be put at the same level of control as players 3,4 , and 5 , which is questionable. Applying the power index $\varphi$ with $\varphi=\Phi^{\omega}, \omega=(1, \ldots, 1) \in \mathbb{R}^{8}$, one obtains

$$
\begin{aligned}
\varphi\left(C^{*}\right) & =(5 / 2,1 / 2,-1,-1,-1) \\
\varphi\left(C^{\prime}\right) & =(3,0,-1,-1,-1) \\
\varphi\left(C^{\prime \prime}\right) & =(4,-1,-1,-1,-1),
\end{aligned}
$$

reflecting each of the three choices above. In favor of $C^{*}$ one could argue that, although player 2 is completely controlled by player 1 , the fact that he has direct control over 3,4 , and 5 , is reflected by its positive power index which, moreover, would increase if the number of players it directly controls, increases. In general, it seems doubtful whether it is possible to find an updating procedure, leading to 'invariant' mutual control structures, that would adequately cover each and every possible situation. In applications, the modelling phase, resulting in a formal mutual control structure $C$, should therefore be done with care.

There are several interesting avenues for further theoretical research in this area. One obvious question is what becomes feasible in terms of power indices if we change the axioms. Section 4.1 already considers some possibilities, e.g., a Banzhaf value based power index becomes possible if the Transfer Property is dropped. Another interesting direction is to consider the combination of mutual control structures with transferable utility games in the spirit of Myerson (1977). A mutual control structure can be seen as a hypergraph, and a value for transferable utility games combined with such a hypergraph can take the imposed control relations into consideration.

\section{References}

Banzhaf JF (1965) Weighted voting doesn't work: a mathematical analysis. Rutgers Law Review 19:317-343

Crama Y, Leruth L (2007) Control and voting power in corporate networks: concepts and computational aspects. European Journal of Operational Research 178:879-893

Crama Y, Leruth L (2011) Power indices and the measurement of control in corporate structures. International Game Theory Review, forthcoming

Dubey P (1975) On the uniqueness of the Shapley value. International Journal of Game Theory 4:131-139 
Dubey P, Einy E, Haimanko O (2005) Compound voting and the Banzhaf index. Games and Economic Behavior 51:20-30

Einy E, Haimanko O (2011) Characterization of the Shapley-Shubik power index without the efficiency axiom. Games and Economic Behavior 73:615621

Gambarelli G, Owen G (1994) Indirect control of corporations. International Journal of Game Theory 23:287-302

Gilles RP, Owen G, van den Brink R (1992) Games with permission structures: the conjunctive approach. International Journal of Game Theory 20:277293

Hu X, Shapley LS (2003a) On authority distributions in organizations: equilibrium. Games and Economic Behavior 45:132-152

Hu X, Shapley LS (2003b) On authority distributions in organizations: controls. Games and Economic Behavior 45:153-170

Myerson RB (1977) Graphs and cooperation in games. Mathematics of Operations Research 2:225-229

Shapley LS (1953) A value for $n$-person games. In: Kuhn HW, Tucker AW (eds) Contributions to the Theory of Games II, Princeton University Press, Princeton.

Shapley LS, Shubik M (1954) A method for evaluating the distribution of power in a committee system. American Political Science Review 48:787-792

Sun H, Driessen T (2006) Semi-marginalistic values for set games. International Journal of Game Theory 34:241-258. 